\title{
Change in dielectric relaxation with the presence of water in highly filled composites
}

\author{
Enis Tuncer \\ Texas Instruments Incorporated, Dallas Texas 75243, USA \\ enis.tuncer@physics.org; e-tuncer@ti.com
}

Received 5 June 2017; Revised 3 October 2017; Accepted 4 October 2017; Published 31 October 2017

\begin{abstract}
It is important to determine the dielectric characteristics of semiconductor encapsulation materials based on epoxy resins. We employed the dielectric spectroscopy technique to investigate the dielectric relaxation in the presence of water and how it changes the relaxation. It was observed that the dielectric relaxation of the material was significantly influenced by absorbed water, the local segmental motion (also known as Johari-Goldstein $(\beta$ ) relaxation) was influenced most by the presence of the water, it was modified by the wet sample compared to dry one, and required high activation energy. The relaxation related to the glass transition was contributed by the cooperative motion (the $\alpha$-relaxation) of the epoxy resin system. The $\alpha$-relaxation was shifted to a low temperature in the wet sample compared to dry one. The relaxation was modeled with a clear Vogel-Fulcher-Tammann-Hesse (VFTH) behavior; the Vogel temperature of the wet sample was $8 \mathrm{~K}$ lower than the dry sample. The presence of water acts as a plasticizer for the molecular relaxation, and speed-up the cooperative process. The measured data were also used to estimate the electrical properties of the resin system by employing an effective-medium model together with a porous media continuum model by taking into account the physical properties of the system. It is already known that the influence of water in semiconductor packaging is important in sensitive applications. The presented measurements and the analysis method would be appreciated within the semiconductor packaging community to improve material selection and performance evaluation efforts.
\end{abstract}

Keywords: Composite materials; semiconductor packaging; dielectric spectroscopy.

\section{Introduction}

Highly filled composites have been used in semiconductor packaging due to their high mechanical modulus to protect the die from environmental stresses. ${ }^{1-4}$ The reason for utilizing high filler contents are three folded; (1) to decrease the mechanical stresses on die; match the mechanical properties of silicon (i.e., thermal expansion stress reduction); (2) to have low enough permittivity and electrical conductivity to control the electric field above the active device with least ionic mobility; and (3) cost; semiconductor grade thermosets resins are expensive and cost reduction is achieved by using high amounts of inorganic fillers (up to $91 \%$ in weight), see Fig. 1. The second item listed above perhaps was not a significant contributor in a material selection process in conventional semiconductor devices, however, with increasing device reliability, sensitivity requirements as well as high voltage applications make the electrical properties of the materials a crucial part of selection criteria. ${ }^{5}$ The properties of the composites need to be known in different conditions to support performance of semiconductor devices. One of those conditions is the influence of moisture on the properties of encapsulation materials. ${ }^{6}$ The presence of water and its influence in the dielectric response were previously studied in various polymeric systems..$^{7-11}$ Here, we concentrate on the change in the dielectric properties of highly filled composites in the presence of water, and report on the dielectric relaxation of moisture-absorbed and dry samples.

\section{Background}

Measurement methods based on change in the electrical impedance have been a valuable tool to investigate material properties. ${ }^{12-19}$ The impedance measurement is a straight forward electrical test once a capacitor structure is built with the material in-hand. There are different commercially available measurement systems that are either called the impedance (dielectric) analyzer or the LCR meter. The method has been applied to various different material systems..$^{711,20-22}$

Electrical properties of materials can be determined from the impedance measurements by performing current measurements with applied voltage and using the geometrical capacitance $C_{0}$, the geometry of the sample. For example, for the complex permittivity, $\varepsilon^{*}$ is estimated from the current measurement as a complex capacitance $C^{*}$ for a given geometrical shape of the measurement electrodes, which determine the geometrical capacitance $C_{0}$,

$$
\begin{aligned}
\varepsilon^{*}(\omega, T) & =C^{*}(\omega, T) / C_{0} \\
& =\varepsilon^{\prime}(\omega, T)-\imath \varepsilon^{\prime \prime}(\omega, T) \\
& =\varepsilon_{\infty}(T)+\chi^{*}(\omega, T)+\sigma_{0}(T)\left(\imath \varepsilon_{0} \omega\right)^{-1} .
\end{aligned}
$$

This is an Open Access article published by World Scientific Publishing Company. It is distributed under the terms of the Creative Commons Attribution 4.0 (CC-BY) License. Further distribution of this work is permitted, provided the original work is properly cited. 


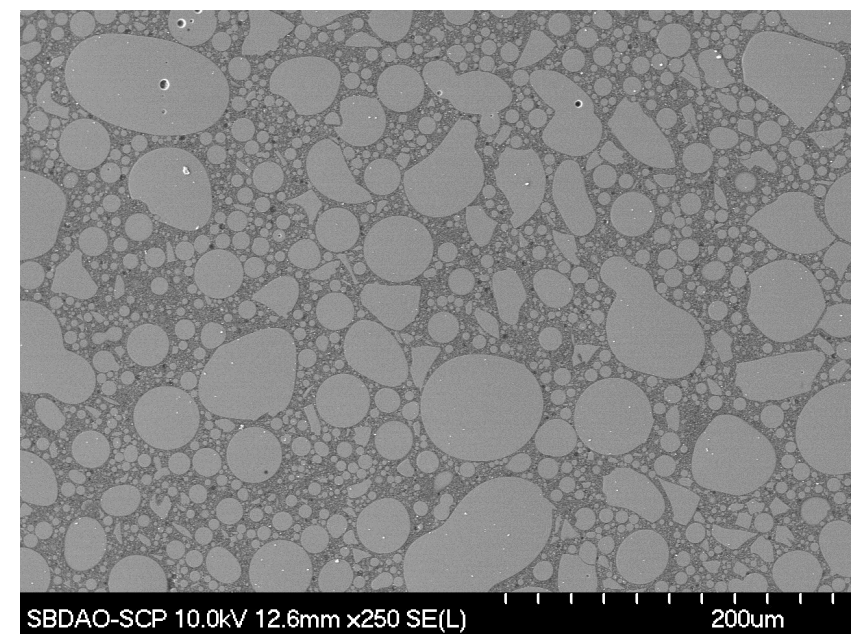

Fig. 1. SEM cross-section image of a mold compound. Observe the high density of mainly spherical filler particles, which are in light gray color.

Here, the angular frequency, $\omega$, independent material properties are of relative permittivity at high frequencies, $\varepsilon_{\infty}$, and Ohmic (direct-current) conductivity, $\sigma_{0} ; l=\sqrt{-1}$.

The frequency-dependent properties related to dipolar polarization and charge transport are included in the dielectric susceptibility, $\chi^{*}(\omega)$. This equation allows us to represent impedance data in different forms; the complex electrical modulus $M^{*}\left(\equiv \varepsilon^{*-1}\right)$; the complex resistivity $\rho^{*}\left(\equiv M^{*}\right.$ $\left.\left(l \omega \varepsilon_{0}\right)^{-1}\right)$ and the complex conductivity $\sigma^{*}\left(\equiv \rho^{*-1}\right)$. The frequency domain measurements require that the impedance is measured at low enough frequencies or high enough temperatures to actually observe the Ohmic losses, ${ }^{17,18}$ however they might be screened with electrode effects, ${ }^{13}$ which is an additional process that needs to be included in the complex susceptibility $\chi^{*}$.

\section{Sample Preparation and Dielectric Measurements}

The reported encapsulation material, also called as the mold compound, in semiconductor packaging here was a mixture of biphenyl and multiaromatic resins with $89.5 \%$ in weight inorganic spherical silica particles. Samples were molded by using the Yamada transfer molding machine, which simulates the actual semiconductor packaging assembly step; the mold kept at $175^{\circ} \mathrm{C}$ and the mold time is $120 \mathrm{~s}$. The dielectric measurements were performed on samples with dimension $25 \mathrm{~mm} \times 25 \mathrm{~mm} \times 0.7 \mathrm{~mm}$. The samples were post-moldcured at $175^{\circ} \mathrm{C}$ for $4 \mathrm{~h}$, which is the conventional cure duration at the assembly and recommended by the supplier. About two different moisture conditions were created using the ambient laboratory $(\sim 50 \% \mathrm{RH})$ condition and the measurement setup, where the dielectric response of samples was measured under $\mathrm{N}_{2}$ atmosphere with a temperature ramp procedure, nonisothermal conditions using several frequencies, as shown in Fig. 2. The data are shown in different frequencies as labeled with logarithm of the measurement frequency on each curve in Fig. 2.

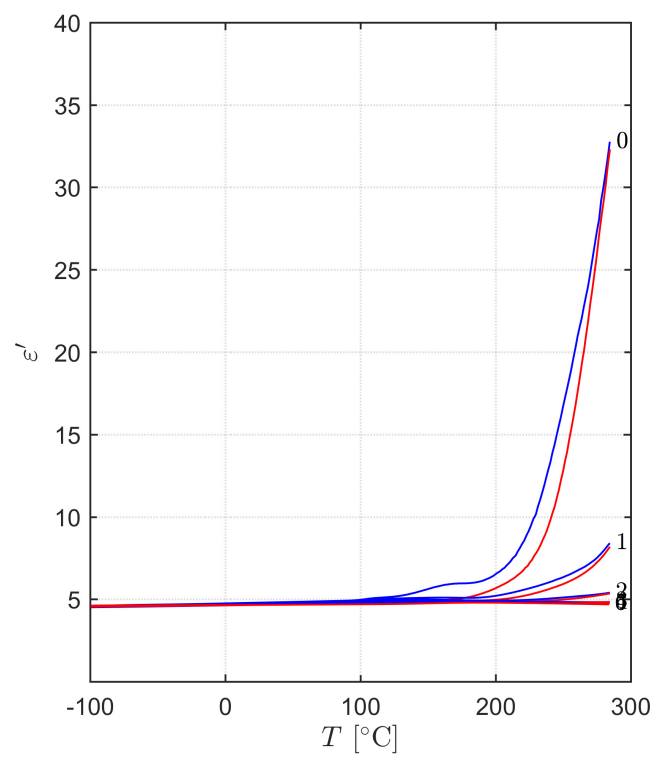

(a)

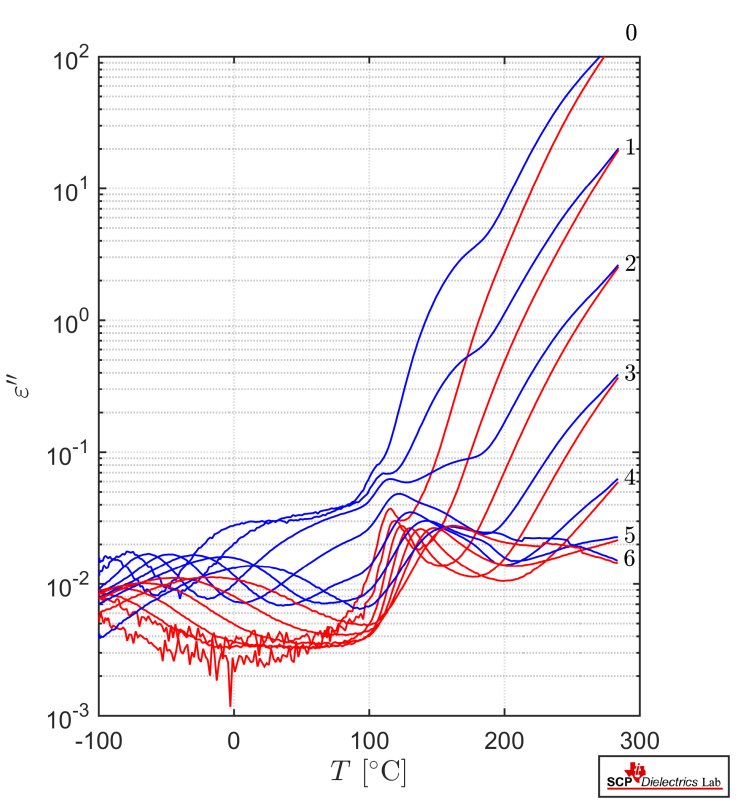

(b)

Fig. 2. (Color online) (a) The real and (b) the imaginary parts of the complex dielectric permittivity of a sample at several frequencies, where the numbers indicate the logarithm of the applied signal frequency. The sample data are shown with blue and red full lines for wet and dry conditions, respectively. The numbers on the right-hand side of each curve indicate the frequency $\nu$ of the measurement in logarithmic scale (e.g., $0 \rightarrow 1 \mathrm{~Hz}$ ). 


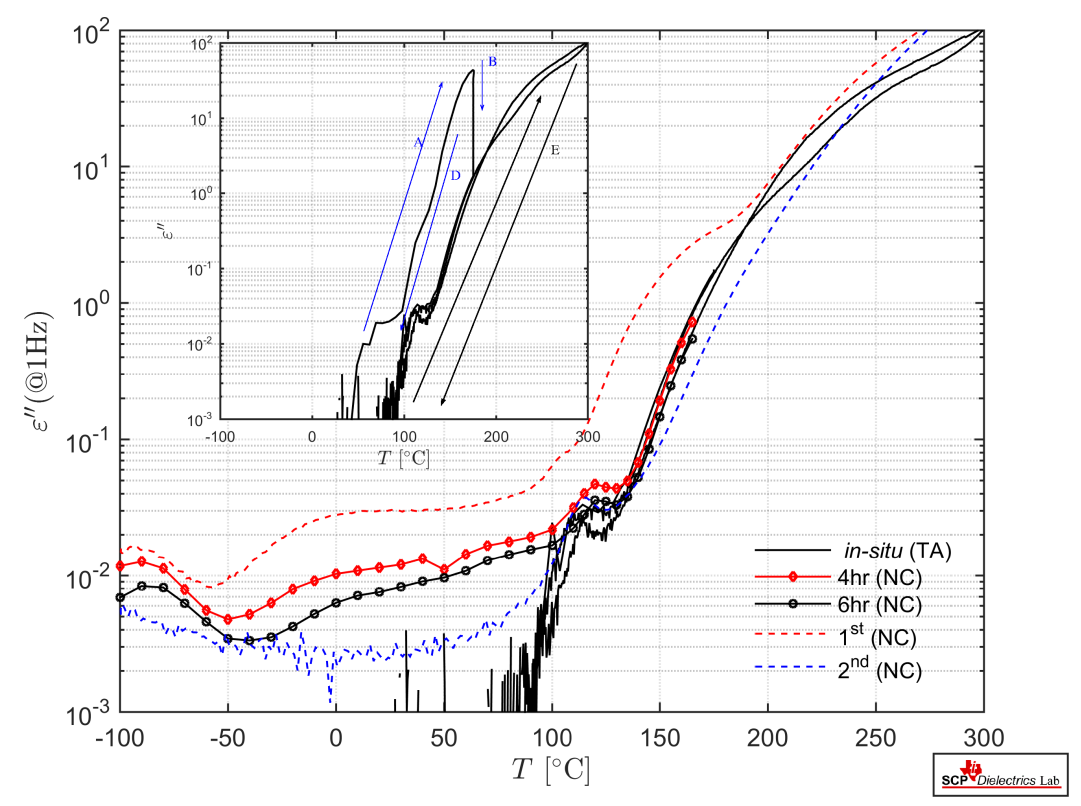

Fig. 3. The imaginary part of the complex dielectric permittivity of samples measured with difference instruments and conditions. The measurements were performed at $1 \mathrm{~Hz}$. The legends TA and NC represent the instruments employed for the measurements, see text for details. The inset shows the in situ post-mold-cure procedure where the sample followed the following processes indicated with arrows and letter labels; (A) rapidly heated to $175^{\circ} \mathrm{C}$ from room temperature, (B) kept at $175^{\circ} \mathrm{C}$ for $4 \mathrm{~h}$ and (D) cooled down to room temperature. To probe the properties after cure cycle, the sample heated to $300^{\circ} \mathrm{C}$ and cooled down back to room temperature as indicated by arrows labeled by $(\mathrm{E})$.

The employed method allows a straightforward characterization of relaxation map in a timely manner and consumes less liquid $\mathrm{N}_{2}$. During the dielectric measurements, the temperature was first cooled down to $-100^{\circ} \mathrm{C}$ and ramped to $285^{\circ} \mathrm{C}$ with $5 \mathrm{~K} / \mathrm{min}$ and second cooled down once again to $-100^{\circ} \mathrm{C}$ and ramped to $285^{\circ} \mathrm{C}$ with $5 \mathrm{~K} / \mathrm{min}$. The water was fully desorbed at the end of the first cycle; our in situ measurements, as shown in Fig. 3 with the solid lines, have no visible peak below $90^{\circ} \mathrm{C}$ in the imaginary part of the complex susceptibility $\chi^{\prime \prime}$, those measurements were performed with DEA2970 Dielectric Analyzer, TA Instruments, USA. The $\alpha$-relaxation (related to the glass transition peak) is clear in the in situ measurements just above $100^{\circ} \mathrm{C}$ in Fig. 3. The other measurements shown in the figure were performed with a Broadband Dielectric Spectrometer from Novocontrol Technologies GmbH \& Co. KG, Germany.

As shown in Fig. 3, the data labeled by $4 \mathrm{~h}$ and $6 \mathrm{~h}$ postmold-cure durations indicate presence of moisture, a significant dielectric loss enhancement between $-10^{\circ} \mathrm{C}$ and $100^{\circ} \mathrm{C}$. Those measurements were performed as the first cycle, and no additional second cycle data were collected. The differences between the first and second measurements under $\mathrm{N}_{2}$ have given us an insight on the influence of the water on the dielectric relaxation, and what it might require to perform measurements to completely disregard the presence of water in the material. It is a dynamic process where the measurement records the diffusion of water molecules during the desorption phase, and data analysis becomes challenging.

\section{Results and Discussion}

\subsection{Dielectric representation}

The differences in the complex susceptibility are significant once the sample is dried, see Fig. 2, where the comparison of the dielectric responses is plotted. The data were further reduced to dielectric susceptibility using Eq. (1), where the high frequency permittivity and conductivity contributions in the permittivity measurement were estimated from high frequency $(1 \mathrm{MHz})$ and $-100^{\circ} \mathrm{C}$, and high temperature $\left(T>270^{\circ} \mathrm{C}\right)$ and low frequency $(1 \mathrm{~Hz})$ data sets, respectively. The estimated susceptibilities are plotted in Fig. 4. Various dielectric activities are present in the samples and some of them are enhanced with the presence of water. The peaks just above $100^{\circ} \mathrm{C}$ are associated to the glass transition of the material, and present in samples no matter whether they are dry or wet. While the low temperature relaxation related to the $\beta$-relaxation has the influence of the water due to differences in the wet and dry sample imaginary parts of the susceptibilities. The high temperature dielectric activity is mostly related to the mobility of ions in the material and they also indicate the presence of the water significantly.

\subsection{Conductivity estimates}

The Ohmic conductivity of the sample was estimated using lowest frequency measurement with the stretched 


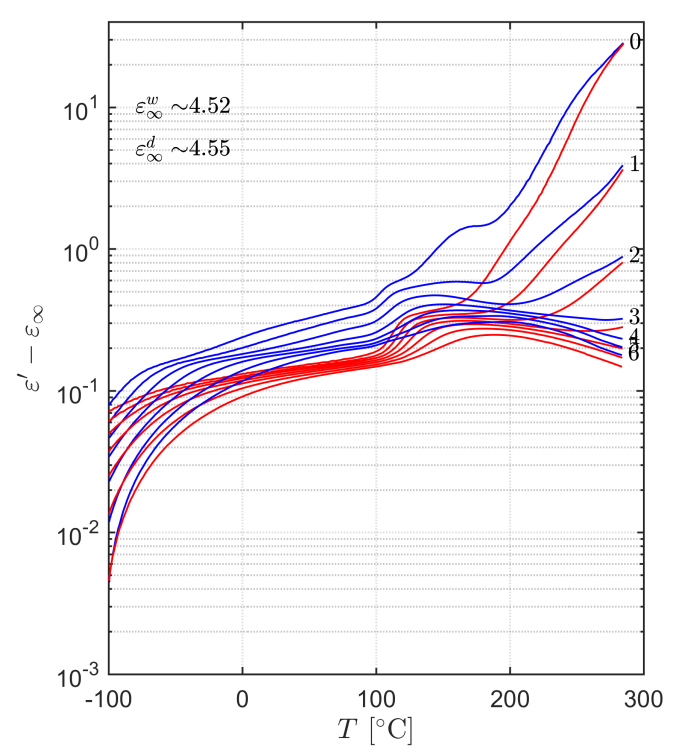

(a)

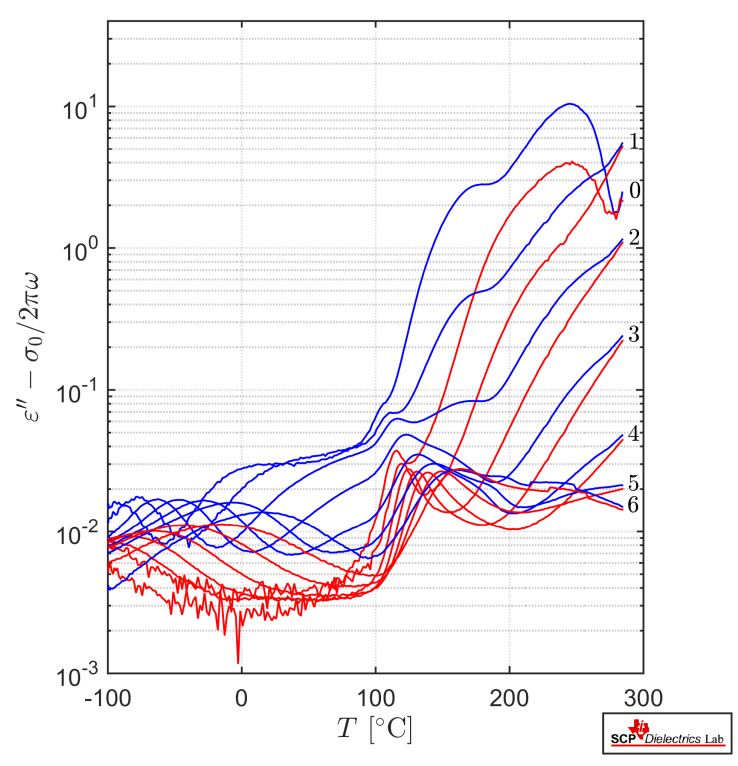

(b)

Fig. 4. (Color online) (a) The real and (b) the imaginary parts of the complex dielectric susceptibility of a sample at several frequencies, where the numbers indicate the logarithm of the applied signal frequency, after isolating the susceptibility $\chi^{*}$ in Eq. (1). The sample data are estimated after subtraction of frequency-independent material properties, the relative dielectric permittivity at high frequencies $\varepsilon_{\infty}$ and Ohmic conductivity $\sigma_{0}$, which is estimated as a function of temperature. The data are shown with blue and red full lines for wet and dry conditions, respectively. The numbers on the right-hand side of each curve indicate the frequency $\nu$ of the measurement in logarithmic scale (e.g., $0 \rightarrow 1 \mathrm{~Hz}$ ).

Table 1. Parameters for the conductivity fits using Eq. (2) and estimated high frequency permittivities.

\begin{tabular}{lcccc}
\hline Sample & $\Sigma[\mathrm{nS} / \mathrm{m}]$ & $T_{0}$ & $\gamma$ & $\varepsilon_{\infty}$ \\
\hline Wet & 338.9 & 766.5 & -4.128 & 4.52 \\
Dry & 320.6 & 728.3 & -4.886 & 4.55 \\
\hline
\end{tabular}

exponential term,

$$
\sigma_{0}=\Sigma \exp \left[\left(T_{0} / T\right)^{\gamma}\right]
$$

where $T$ is the absolute temperature, $\Sigma, T_{0}$ and $\gamma$ are fit parameters; the fit results are tabulated in Table 1. A stretch exponential instead of a exponential term $(\gamma=1)$ is adapted because the current material system is a polymeric system, which often illustrates an ionic conduction behavior observed at temperatures over the glass transition temperature $T_{g}$, which also indicates the start of the cooperative motion of the polymer chains $-\alpha$-relaxation. ${ }^{23,24}$ The reason for using the $1 \mathrm{~Hz}$ data can be observed in Fig. 5, where the real part of the complex conductivity is converging to one line for low frequency measurements; details of the procedure are described by the author elsewhere. ${ }^{17,18}$ There is slight difference in the conductivities at high temperatures, however the low temperature conductivity contribution is significant as expected in the wet sample.

\subsection{Dielectric relaxations}

We treat the first measurement as a dry-bake process to desorp the water; the heating rate was $5 \mathrm{~K} / \mathrm{min}$ and the change in the dielectric response, as shown later in the paper, becomes troublesome to explain during the desorption period. The data over $100 \mathrm{~Hz}$ illustrate the decrease in the real part of the susceptibility over $140^{\circ} \mathrm{C}$, see Figs. 4 and 6, which could be regarded as the desorption event; susceptibility should increase with temperature due to the nature of the material, there are no ferroelectric transitions to cause decrease in the real part of the susceptibility. ${ }^{20-22,25}$

The number of relaxations that can be observed in wet sample is larger than the dried sample. As discussed previously, at high temperatures close to $300^{\circ} \mathrm{C}$, the data for the wet and dried sample are converging to each other indicating the similarities in both responses and confirm that the initial measurement in the first ramp is a dry-bake cycle. The relaxations related to the molecular nature of the material are associated in the dried sample, which indicated three significant processes; (i) a low temperature relaxation attributed to the local (segmental) molecular motion often called as the Johari-Goldstein $(\beta)$ relaxation; ${ }^{26-28}$ (ii) the glass-transition related loss from cooperative $(\alpha)$ relaxation at mid-temperatures; ${ }^{28}$ (iii) a relaxation related to the conduction losses and Maxwell-Wagner-Sillar (MWS, also known as the interfacial relaxation $)^{29-38}$ at high temperatures. The high temperature process overlaps with the 


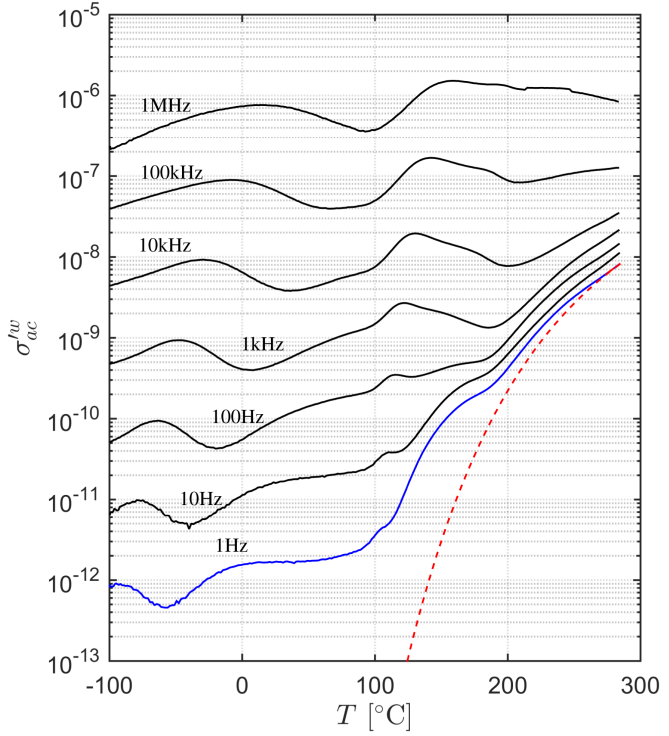

(a)

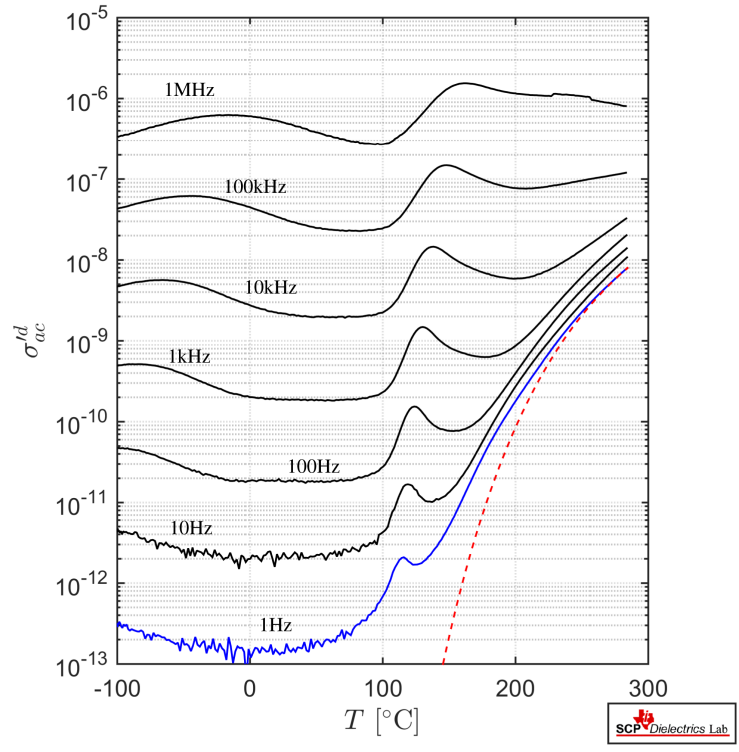

(b)

Fig. 5. The real part of complex conductivity of a sample at (a) wet and (b) dry conditions. The lowest frequency data (1 Hz) are the bottom curves in both the conditions. The curve fits for the stretched exponential term in Eq. (2) using the high temperature part of the data are plotted with dashed lines. The frequency of the measurement are labeled on the curves.

conductive losses because of its nature; it occurs at the interface boundary between constituents in composites. When the matrix has a higher conductivity than the inclusions, the interfacial polarization losses are hidden under the conductive (Ohmic) losses. The composite system studied here is a good example for such dielectric response since the dielectric permittivity and the electrical conductivity of the resin are higher than the employed inorganic silica filler particles.

\subsection{Dielectric relaxation map}

Two different approaches are considered to build the relaxation map of the sample (i) straightforward maximum loss

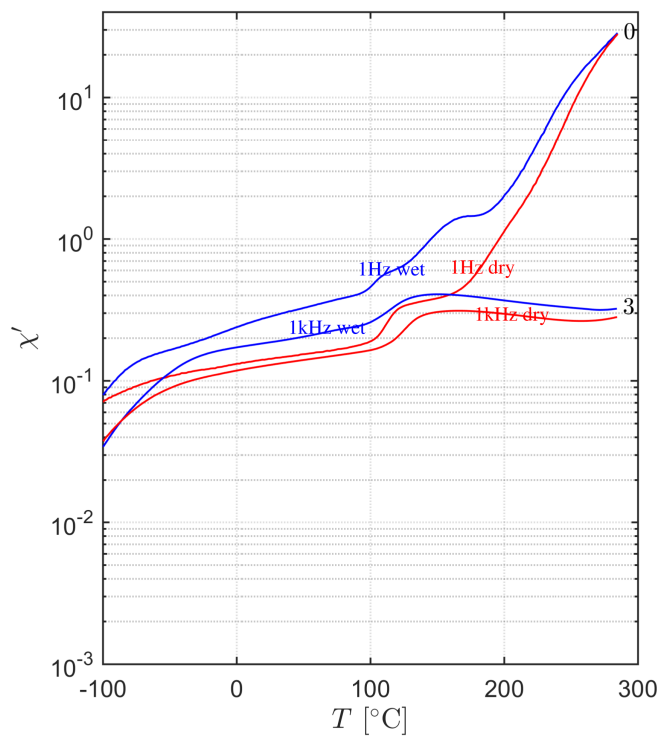

(a)

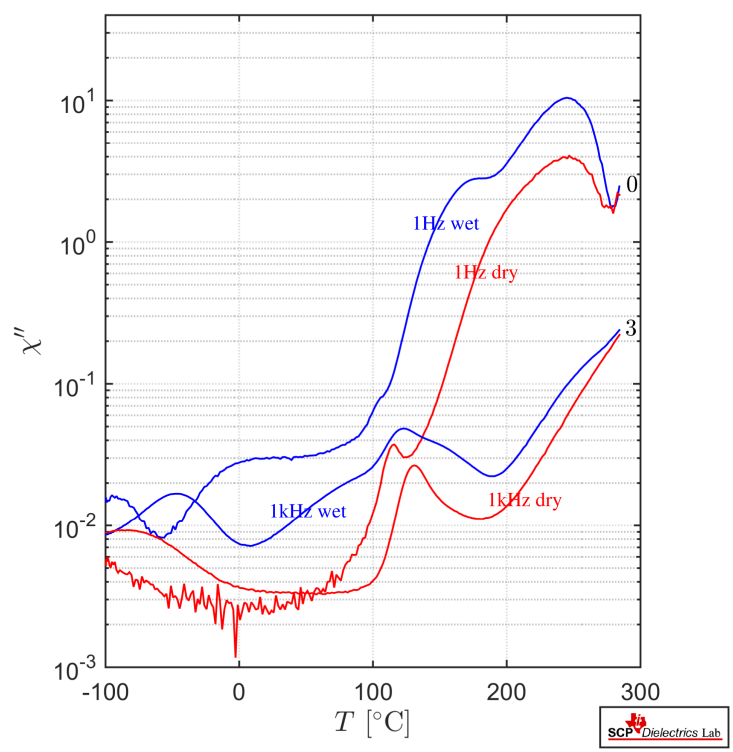

(b)

Fig. 6. The real (a) and the imaginary (b) parts of the complex dielectric susceptibility of a sample at two different frequencies; $1 \mathrm{~Hz}$ and $1 \mathrm{kHz}$, labeled with 0 and 3, respectively. The first and the second measurement runs are shown to illustrate the changes in the dielectric response, where the presence of water results in higher polarization activity observable in the real and the imaginary parts of the susceptibility compared to dried sample. 


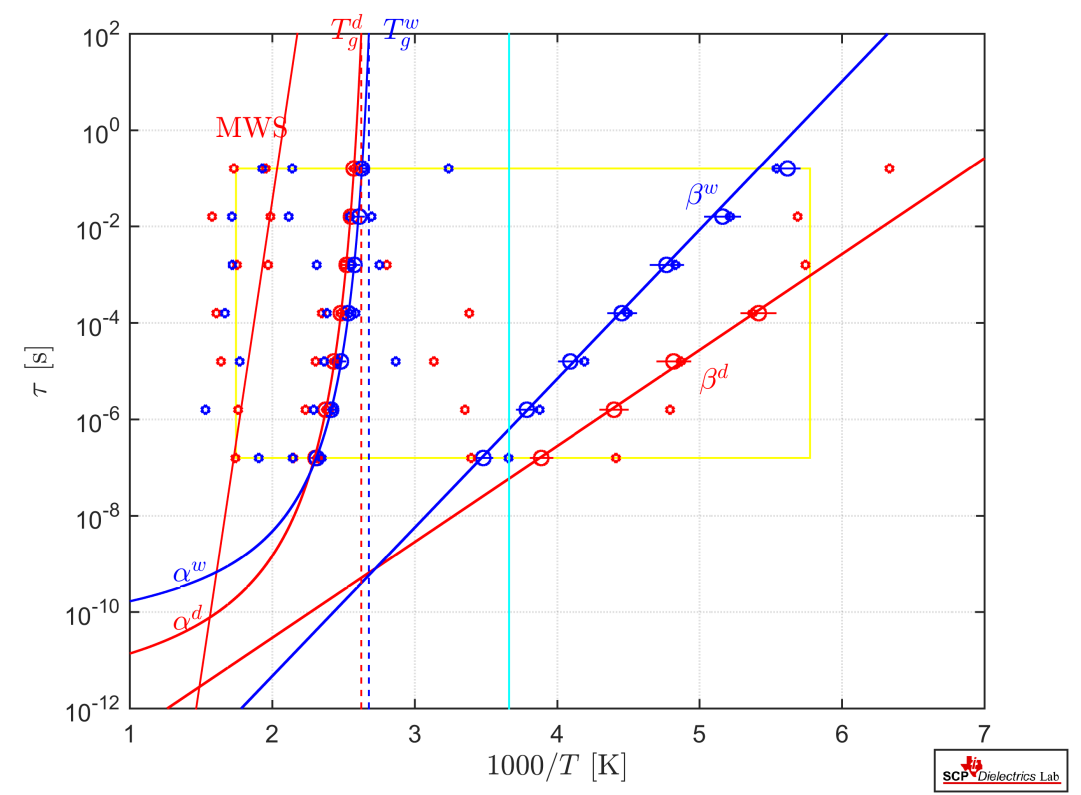

Fig. 7. The relaxation map of the wet and dry samples. Different relaxation processes are marked on the plot. The glass transition temperature for the sample at different conditions are marked with the vertical dashed lines and positions shown with $T_{g}$ label at $100 \mathrm{~s}$ intersection points of the vertical lines. The box indicates the experimental window, which is the region probed with the measurement conditions. The star ( $\star$ ) symbols are obtained from the Lévy distribution fits using Eq. (3) to the imaginary part of the susceptibility.

temperature locations $\bar{T}$ (ii) nonlinear curve fitting with Lévy distribution, ${ }^{36,39-42}$ which allow the deconvolution of the measured peaks,

$$
\begin{aligned}
\mathfrak{L}(T ; A, \bar{T}, z, s, \gamma)= & \mid A \exp \left\{\imath T s-z^{\gamma}|T-\bar{T}|^{\gamma}\right. \\
& \times[1+\imath s \tan (\pi \gamma / 2) \operatorname{sgn}(T)]\} \mid .
\end{aligned}
$$

Here, $0<\gamma, 2$. We need only four parameters: $\gamma$, the characteristic exponent, $\bar{T}$, the localization parameter, $z$, the scale parameter and $A$, the amplitude, $s$ is considered zero since there is no negative susceptibility, which has no significance for the results. The special forms of Eq. (3) are the Gaussian $[\mathfrak{L}(T ; A, \bar{T}, 2, z)]$, the Lorentz or Cauchy $[\mathfrak{L}(T ; A, \bar{T}, 1, z)]$ and the Gamma $[\mathfrak{L}(T ; A, \bar{T}, 1 / 2, z)]$ distributions. Different forms of probability density functions for Levy statistics exist; we have adopted a stable distribution used by Breiman, ${ }^{41}$ Loeve ${ }^{39}$ and Walter. ${ }^{42}$ Except for the localization parameter of the Levy distributions, the others are disregarded in this study.

The estimated values of $\chi^{\prime \prime}$ in Fig. 4(b) were used in Eq. (3), $\chi^{\prime \prime}(T)=\mathfrak{L}(T ; A, \bar{T}, z, 0, \gamma)$, such that the temperature localization parameter $\bar{T}$ is used to assign the relaxation time for a given process. The properties of the Lévy distribution are summarized elsewhere. ${ }^{36}$ Except for the localization parameter of the Lévy distributions, the others are disregarded in this study. An example of the analysis is shown in Fig. 8, where the peaks of different relaxation processes identified for the data are obtained at $1 \mathrm{kHz}$.

The first approach yields the relaxation map shown in Fig. 7, where the actual location of the peaks observed is

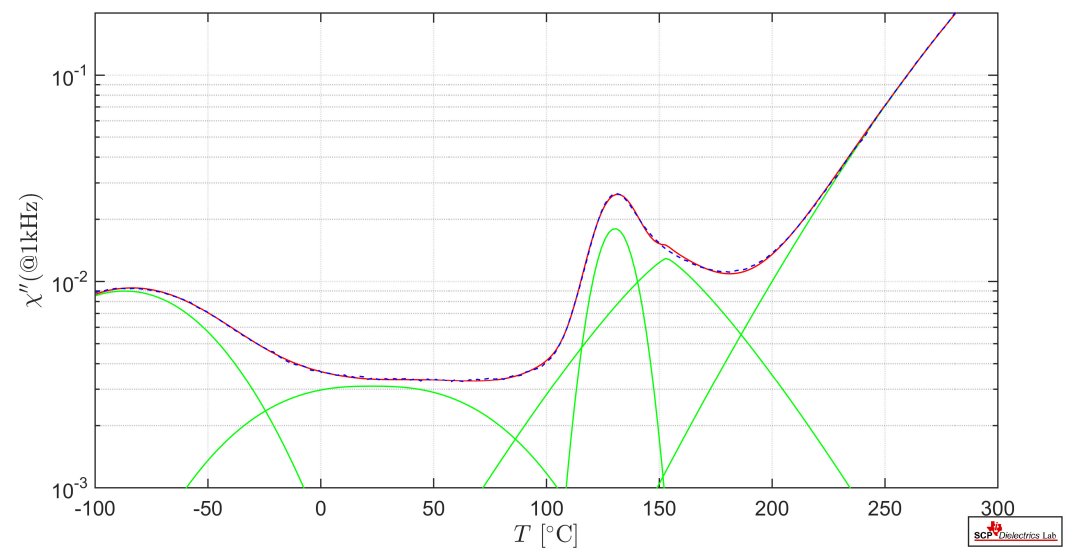

Fig. 8. Response at $1 \mathrm{kHz}$ and the fitted Lèvy distributions, where the susceptibility was deconvoluted to several different peaks with Eq. (3). 
Table 2. Parameters for the $\alpha-, \beta$ - and $a$-relaxations using Arrhenius and VFTH equations.

\begin{tabular}{lccccccc}
\hline Sample & Relaxation & Eq. & $\tau_{0}[\mathrm{~s}]$ & $T_{0}$ & $T_{V}[\mathrm{~K}]$ & $T_{g}[\mathrm{~K}]$ & $T_{g}\left[{ }^{\circ} \mathrm{C}\right]$ \\
\hline \multirow{2}{*}{ Wet } & $\beta$ & $(4)$ & $3.191 \times 10^{-18}$ & 7104 & - & - & - \\
& $\alpha$ & $(5)$ & $6.131 \times 10^{-11}$ & 651.3 & 350.4 & 373.5 & 100.4 \\
Dry & $\beta$ & $(4)$ & $3.069 \times 10^{-15}$ & 4582 & - & - & - \\
& $\alpha$ & $(5)$ & $3.463 \times 10^{-12}$ & 894.8 & 352.2 & 381.1 & 108.0 \\
& MWS & $(4)$ & $1.261 \times 10^{-41}$ & $4.541 \times 10^{4}$ & - & - & - \\
\hline
\end{tabular}

shown with open symbols. The presence of the three relaxations is visible in the dry sample. The local segmental motion from the resin system is observed at low temperatures, and it is Arrhenius activated,

$$
\tau=\tau_{0} \exp \left[T_{0} T^{-1}\right]
$$

The fit parameters for Eq. (4) are listed in Table 2. The $\beta$-relaxation does not indicate a significant deviation from Arrhenius behavior. It is interesting to see that the $\beta$ relaxation in the wet sample was different and shifted up in the relaxation map. This was an indication for a requirement of a higher energy for segmental motion than the dry sample. It is clear that the presence of water within the resin causes the segmental motion to move slower. If this relaxation is assumed to be a new one, then it can be associated to the water dynamics around the segmental motion which gives rise to the dielectric losses in the wet sample. However, this argument can be supported. Because it is remarkable that the $\beta$-relaxation behavior for the wet and dry conditions intersect at around glass transition for both cases indicating that the origin of the process is actually connected to the cooperative motion ( $\alpha$-relaxation), in such a way that as we reach the glass transition temperature, the large-scale cooperative relaxations free the water molecules. One can conclude that the water is absorbed locally and due to the behavior of the segmental relaxation, it is not in large pockets; it influences the microscopic motion of the resin system; this would be an indication of short range order and influence of the water molecules in short ranges.

The relaxation map plot of the $\alpha$-relaxation is significantly bent, an indication of the co-operative behavior and it is connected to the glass transition temperature, since it appears around the glass transition. It was modeled with VogelFulcher-Tammann-Hesse (VFTH) expression, ${ }^{43-45}$

$$
\tau=\tau_{0} \exp \left[T_{0}\left(T-T_{V}\right)^{-1}\right]
$$

with $\tau_{0}, T_{0}$ and the Vogel temperature $T_{V}$ being curve fit parameters. The Vogel temperature is known to be closely related to the dynamic glass transition temperature $T_{g}$ which is usually defined as the temperature where the relaxation time is $100 \mathrm{s.} .^{28,46}$ The VFTH fit parameters $\tau_{0}, T_{0}$ and $T_{V}$ of the relaxation as well as the glass transition temperature $T_{g}$ are listed in Table 2 for the two conditions. It is clear that the water influences the cooperative process and the glass transition estimated from the VFTH is around $8 \mathrm{~K}$, higher in dry sample compared to the wet one. The presence of water creates some lubrication as a plasticizer for the cooperative motion, and molecular relaxations become slightly fast due to the change in the behavior around the glass transition. The water can be absorbed in the mold compound in different locations such as the interface between the filler particles and resin and within the resin, which is a polymeric network. Going back to the present water locally inside the polymer chains, it is expected that the shape of the cooperative motion would not be influenced with water molecules; the system continues to behave with long range correlation. However, the shift in the glass transition temperature of the wet resin system shows a confinement effect as discussed by Arndt et $a l .{ }^{47}$ for salol relaxation dynamics in nanometer size pores; the relaxation rate of the confined material increased compared to bulk material.

Some other examples of observed low $T_{g}$ with moisture/ water content can be found in the literature, ${ }^{10,11,48}$ where change in the glass temperature was reported for inulin, epoxy resins and cellulose. The epoxy system studied ${ }^{11}$ showed that the glass transition estimated from the mechanical modulus shifted to lower temperatures with different moisture treatment with shift related to the moisture weight gain, which we have not included in this work. Similarly, the work on the cellulose ${ }^{10}$ indicated similar observations as the epoxy system. Comparing the current results with those reports, it can be concluded that the water absorption was not as significant as those cases, which had larger $T_{g}$ shifts than the current work.

The MWS relaxation was clear in the wet sample, however it did not show a clear temperature dependence, we attribute this to the desorption kinetics, since the sample was in a condition with changing water content and in diffusion phase. The temperature for this relaxation was higher than the other two and therefore the water molecules would have enough energy to diffuse and generate a more complex relaxation scene. However, when the sample was dried, the relaxation is just under the Ohmic losses, as expected. It did have a Arrhenius-type behavior as shown in Fig. 7 and Table 2.

The fits from the second approach are also shown in Fig. 7 with star $(\star)$ symbols. There is a nice overlap with the straightforward method as one expects. However, there are several additional relaxations as shown for the wet sample at high temperatures which is due to the MWS and influence of water on that phenomenon. 


\subsection{Dielectric mixture approach}

We include an additional analysis based on the theory of dielectric mixtures. ${ }^{37,38,49-51}$ Here, we assume that the electrical properties of the studied composite system (labeled " $c$ ") could be represented with a binary mixture approach where two phases are the resin (labeled " $r$ ") and inorganic filler particles (labeled " $f$ "). Once we adopt this approach, the electrical properties of the highly filled composite can be expressed with the scaled complex permittivity $\xi$ approach as follows: ${ }^{50-55}$

$$
\xi(\varpi)=\xi_{p}+\xi_{n}\left[1+(\varpi x)^{a}\right]^{-b},
$$

where $\xi=\left(\varepsilon_{c}^{*}-\varepsilon_{r}^{*}\right)\left(\varepsilon_{f}^{*}-\varepsilon_{r}^{*}\right)^{-1}$ and $\varpi$ is the spectral frequency and $x$ is the spectral parameter. The parameters $a$ and $b$ are free parameters to generate different types of effective permittivity values. The constant parameters $\xi_{p}$ and $\xi_{n}$ are the percolating and non-percolating volume fraction of the filler inclusions. Equation (6) would yield to the bounds in mixtures with different values of $x ; x=0$ would be the percolating condition and $x=1$ would be the insulating condition for $a=b=1$, which are the Wiener ${ }^{56}$ bounds. The details of the spectral density model and other bounds can be found in the literature. ${ }^{57-68}$ The numerical examples have been published by the current author. ${ }^{50-54}$ One of the Wiener bounds is as follows, which is the geometrical addition of the constituent properties to represent the composite effective property, here, $q$ is the volume fraction of the filler phase:

$$
\begin{gathered}
\varepsilon_{c}=(1-q) \varepsilon_{r}+q \varepsilon_{f}, \\
\sigma_{c}=(1-q) \sigma_{r}+q \sigma_{f} .
\end{gathered}
$$

The filler $\left(\mathrm{SiO}_{2}\right)$ used in the present system is highly insulating compared to the resin, therefore it is more suitable

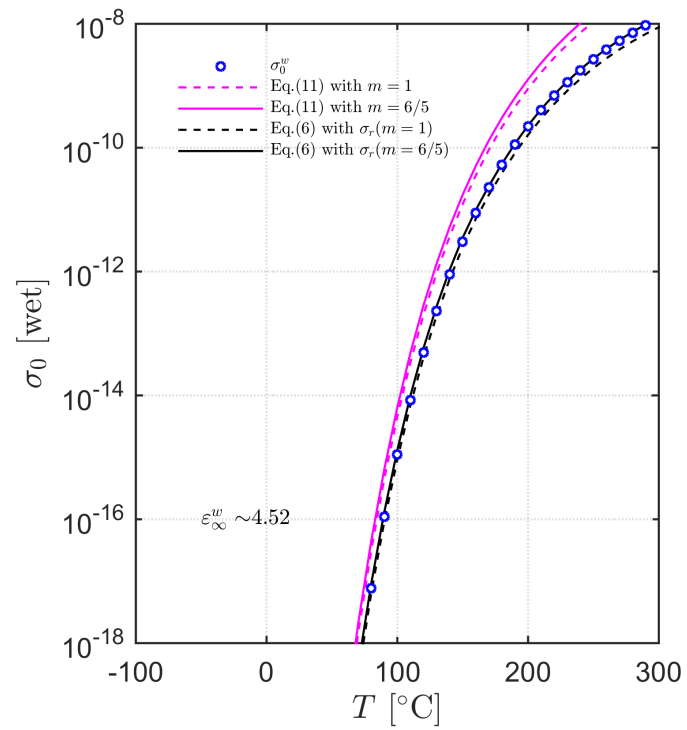

(a) to study the conductivity of the system compared to the permittivity. The conductivity expression can be represented by Archie's law ${ }^{69-75}$ instead (similar approaches for porous system mechanical properties have also been proposed ${ }^{76}$ ). This approach is valid for the current system since the insulating nature of the filler particles and the electrical current are mainly conducted through the resin system, see Nettelblad et al $^{74,75}$ The Archie's law or relation is as follows: ${ }^{69}$

$$
F \propto q^{-m} \quad \text { with } \quad F \equiv \sigma_{c} / \sigma_{r}
$$

where the parameter $F$ is the formation factor and is just the ratio of the effective composite property to that of the conducting phase; the parameter $m$ is called the cementation index and it takes values between 1 and 5, ${ }^{70,73}$ and it would depend on the topology of the mixture. Equation (9) can be used as an effective medium model for cases when the electrical current is fully confined in the pores or as in our case in the ionically conducting resin, such that

$$
\sigma_{c}=\sigma_{r}(1-q)^{-m} \text {. }
$$

The following procedure was employed to estimate and verify the conductivity of the resin for both wet and dry conditions; (i) using Eq. (10), the cementation value $m$ was assigned as a free parameter; (ii) $\sigma_{r}$ was estimated using the $\sigma_{0}^{w}$ and $\sigma_{0}^{d}$ from Table 1 ; (iii) $\sigma_{r}$ was later inserted in the complex permittivity of the resin in Eq. (1) with resin permittivity as a free parameter as well; (iv) the permittivity of the filler $\varepsilon_{f}$ is taken to be the relative permittivity of $\mathrm{SiO}_{2}$, $\varepsilon_{f} \equiv 3.9$; (v) the low temperature permittivity of the composite and the temperature-dependent conductivity of the composite were used to get the best fit parameters for

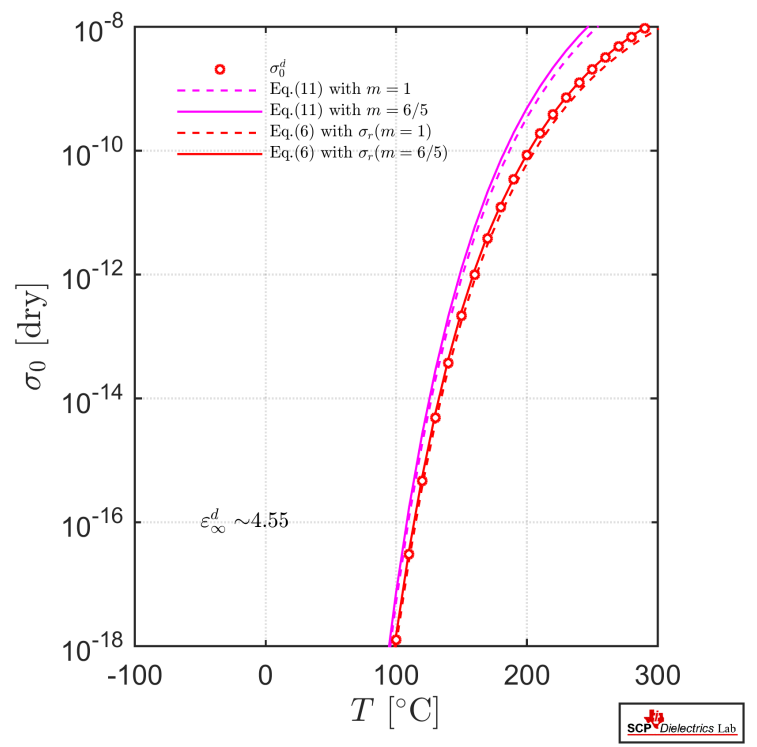

(b)

Fig. 9. The conductivity models for (a) dry and (b) wet conditions. Observe that Eq. (10) with $m=1$ becomes similar to Eq. (7) for the estimate of the conductivity for the resin. 
$m, \varepsilon_{r}$ and $\sigma_{r}(T)$. The concentration of the resin was calculated from weight percentages of the filler and resin components using densities $\rho_{\mathrm{SiO}} x=2.65 \mathrm{~g} / \mathrm{cc}$ and $\rho_{r}=1 \mathrm{~g} / \mathrm{cc}$ and filler weight fractions $0.89 \mathrm{wt} . \%$, yielding $q=0.75$.

The spectral parameter in Eq. (6) was assigned to $x=(1-q) / 3$, which is for spherical inclusions, and for straight forward approach, $a=b=1$ was considered without introducing any filler size and shape distributions. Best estimates were obtained with $m \sim 5 / 4$, and $\varepsilon_{r}^{w}=6.70$ and $\varepsilon_{r}^{d}=6.85$, see Fig. 9. For $m=1$, the Archie's Law is the same as Eq. (7), which is also shown in Fig. 9. The modeled data for the conductivities matched closely to the conductivities estimated from the measurement data shown in Fig. 5 with the dashed lines. The obtained permittivity values are a little higher than expected, however, the initial relative permittivity measurements at time zero indicated a value around 4.5 , which influence the high permittivity results in the model.

No attempt to model the complex susceptibility is performed in this paper due to difficulties in determining or lack of the electrical properties of the pure, unfilled resin system. One can employ a similar procedure as explained in, ${ }^{37,51-54,77-80}$ however the kernel for the Fredholm integral $^{81}$ needs to be altered to a temperature-dependent function.

\section{Conclusions}

The analysis of the dielectric measurements, which were performed using a isochronal procedure, provided some insight regarding the influence of water in the dielectric relaxation dynamics in highly filled composite materials for semiconductor packaging. The results indicated that the presence of the water significantly influenced the local segmental motion of the resin (polymeric) system, while the co-operative motion connected to the glass transition was slightly shifted both the temperature dependence of the relaxation and the glass transition temperature. Some of the practical implications of the water in the composite system are the increase in the dielectric losses and also decrease in the $T_{g}$ of the composite system. The latter one would influence the thermo-mechanical performance and the former one would influence the high voltage and electrical lifetime performance of the materials such that one should expect quick degradation under voltage applied conditions. The application of the effective-medium approach to the electrical properties of the system provided predictions for the physical nature of the conduction and polarization in these highly filled resin system. Note that it is often impractical or extremely hard to study pure constituent properties, unfilled samples, because of the preparation procedures for semiconductor encapsulation compounds used in transfer molding process.

Finally, we would like to draw attention to the potential of the temperature-dependent dielectric spectroscopy measurements in highly filled composites. Molecular relaxation dynamics of a system can provide valuable information. It can be employed to map relaxations and determine, if any, the influence of water on the material additional to conventional approaches. ${ }^{6}$ There is a need to study the behavior of water in similar systems to better understand the material used in semiconductor packaging.

\section{Acknowledgments}

The author would like to thank the Texas Instruments Taiwan Semiconductor Packaging team (TITL SCP) for providing an image of one of the materials used in packaging. I would also like to thank the reviewers for pointing out the relaxation dynamics and absorbed water.

\section{References}

${ }^{1}$ National Research Council, Materials for High-Temperature Semiconductor Devices (The National Academies Press, Washington, DC, 1995), doi: 10.17226/5023.

${ }^{2}$ A. Chen, A. Shafi, R. Busse, R. Orr and R. Lo, Molding compounds for high breakdown voltage applications on power ic semiconductors, 47th Proc. Electronic Components and Technology Conf. (1997), pp. 91-94.

${ }^{3}$ D. Lu and C. Wong, Materials for Advanced Packaging (Springer, Berlin, 2009).

${ }^{4}$ P. Gonon, A. Sylvestre, J. Teysseyre and C. Prior, Dielectric properties of epoxy/silica composites used for microlectronic packaging, and their dependence on post-curing, J. Mater. Sci. Mater. Electron. 12, 81 (2001).

${ }^{5}$ E. Tuncer, The glass transition and dc conductivity in highly filled composites, Mater. Res. Express 4, 045905 (2017).

${ }^{6}$ X. J. Fan and E. Suhir (eds.), Moisture Sensitivity of Plastic Packages of IC Devices, (Springer, Berlin, 2010).

${ }^{7}$ J. Mijovi and H. Zhang, Local dynamics and molecular origin of polymer network-water interactions as studied by broadband dielectric relaxation spectroscopy, ftir, and molecular simulations, Macromolecules 36, 1279 (2003).

${ }^{8}$ Y. Feldman, T. Skodvin and J. Sjöblom, Encyclopedic Handbook of Emulsion Technology, Chapter 6, ed. J. Sjöblom (Marcel Dekker, New York, 2001), pp. 109-168.

${ }^{9}$ E. Laredo, M. Grimau, F. Snchez and A. Bello, Water absorption effect on the dynamic properties of nylon- 6 by dielectric spectroscopy, Macromolecules 36, 9840 (2003).

${ }^{10}$ L. Szcześniak, A. Rachocki and J. Tritt-Goc, Glass transition temperature and thermal decomposition of cellulose powder, Cellulose 15, 445 (2008).

${ }^{11}$ A. Apicella, L. Nicolais, G. Astarita and E. Drioli, Effect of thermal history on water sorption, elastic properties and the glass transition of epoxy resins, Polymers 20, 1143 (1979).

${ }^{12}$ J. R. Macdonald (ed.), Impedance Spectroscopy (John Wiley \& Sons, New York, 1987).

${ }^{13}$ E. Barsoukov and J. R. Macdonald (eds.), Impedance Spectroscopy: Theory, Experiment and Applications, 2nd edn. (John Wiley \& Sons, New York, 2005).

${ }^{14}$ F. Kremer and A. Schönhals (eds.), Broadband Dielectric Spectroscopy (Springer-Verlag, Berlin, 2003). 
${ }^{15}$ E. Tuncer, I. Sauers, D. James and A. R. Ellis, Electrical properties of a commercial resin, Conf. Electrical Insulation and Dielectric Phenomena (CEIDP'06) Annual Report (2006), pp. 117-120.

${ }^{16}$ E. Tuncer, I. Sauers, D. James, A. Ellis, G. Polizos and M. Pace, Single frequency characterization of a commercial resin, IEEE Conf. Electrical Insulation and Dielectric Phenomena, 2009 (CEIDP '09) (2009). pp. 158-160.

${ }^{17}$ E. Tuncer, Discussion on impedance measurements and bulk ohmic conductivity in lossy dielectrics, 2014 IEEE Conf. Electrical Insulation and Dielectric Phenomena (CEIDP) (2014), pp. 844-847.

${ }^{18}$ E. Tuncer, Comparison of conductivities obtained from dielectric spectroscopy and time domain measurements, 2016 IEEE Conf. Electrical Insulation and Dielectric Phenomena (CEIDP) (2016), pp. 768-770.

${ }^{19}$ K. U. Kirsh, F. Kremer and V. M. Litvinov, Broad-band dielectric spectroscopy on the molecular dynamics of bulk and absorbed poly(dimethylsiloxane), Macromolecules 26, 975 (1993).

${ }^{20}$ H. S. Nalwa (ed.), Ferroelectric Polymers (Marcel Dekker Inc., New York, 1995).

${ }^{21}$ T. Wang, J. Hu, H. Yang, L. Jin, X. Wei, C. Li, F. Yan and Y. Lin, Dielectric relaxation and maxwell-wagner interface polarization in nb2o5 doped 0.65bifeo30.35batio3 ceramics, J. Appl. Phys. 121, 084103 (2017).

${ }^{22}$ T. Wang, L. Jin, C. Li, Q. Hu and X. Wei, Relaxor ferroelectric batio3bi(mg2/3nb1/3)o3 ceramics for energy storage application, J. Am. Ceram. Soc. 98, 559 (2015).

${ }^{23}$ E. Tuncer, M. Wegener and R. Gerhard-Multhaupt, Distribution of relaxation times in $\alpha$-phase polyvinylidene fluoride, J. Non-Cryst. Solids 351, 2917 (2005).

${ }^{24}$ E. Tuncer, M. Wegener, P. Frübing and R. Gerhard-Multhaupt, Origin of temperature-dependent conductivity in $\alpha$-polyvinylidene fluoride, J. Chem. Phys. 122, 084901 (2005).

${ }^{25}$ R. G. Kepler, Ferroelectric Polymers: Chemistry, Physics and Applications, Chapter 3, ed. H. R. Nalwa (Marcel Dekker, New York, 1995).

${ }^{26}$ J. G. McAnanama, D. A. Wasylyshyn and G. P. Johari, Temperature-independent onset of diffusion control during polymerization in a diepoxide-amine mixture by dielectric measurements, Chem. Phys. 252, 237 (2000).

${ }^{27} \mathrm{G}$. P. Johari, Dielectric relaxations in the liquid and glassy states of poly(propylene oxide) 4000, Polymers 27, 866 (1986).

${ }^{28}$ E. Donth, The Glass Transition: Relaxation Dynamics in Liquids and Disordered Materials, (Springer-Verlag, Berlin, 2001).

${ }^{29}$ J. C. Maxwell, A Treatise on Electricity and Magnetism - Volume 1, 3rd edn. (Clarendon Press, Oxford, 1891), pp. 450-464.

${ }^{30} \mathrm{~K}$. W. Wagner, Erklärung der dielekrischen Nachwirkungsvorgänge auf Grund Maxwellscher Vorstellungen, Arch. Electrotechn. II, 371 (1914).

${ }^{31}$ K. W. Wagner, Zur theorie der unvollkommenen dielektrika, Ann. Phys. 40, 817 (1913).

${ }^{32} \mathrm{R}$. Sillars, The properties of a dielectric containing semiconducting particles of various shapes, J. Inst. Electr. Eng. 80, 378 (1937).

${ }^{33}$ R. W. Sillars, Electrical Insulating Materials and their application (Peter Peregrinus Ltd. on behalf of the Institution of Electrical Engineers, Stevenage, England, 1973).

${ }^{34} \mathrm{H}$. Fricke, The Maxwell-Wagner dispersion in a suspension of ellipsoids, J. Phys. Chem. 57, 934 (1953).

${ }^{35} \mathrm{H}$. Fricke, A mathematical treatment of the electric conductivity and capacity of disperse systems, Phys. Rev. 24, 575 (1924).
${ }^{36}$ E. Tuncer, Signs of low frequency dispersions in disordered binary dielectic mixtures (fifty-fifty), J. Phys. D, Appl. Phys. 37, 334 (2004).

${ }^{37}$ E. Tuncer, Dielectric mixtures-importance and theoretical approaches, IEEE Electr. Insul. Mag. 29, 49 (2013).

${ }^{38}$ E. Tuncer, Y. V. Serdyuk and S. M. Gubanski, Dielectric mixtures: Electrical properties and modeling, IEEE Trans. Dielectr. Electr. Insul. 9, 809 (2002).

${ }^{39}$ M. Loéve, Probability Theory I, 4th edn. (Springer-Verlag, Berlin, 1977).

${ }^{40}$ W. Feller, An Introduction to Probability Theory and Its Applications (John Wiley and Sons, New York, 1970).

${ }^{41}$ L. Breiman, Probability (Addison-Wesley, Reading, 1968).

${ }^{42} \mathrm{C}$. Walter, Lévy-stability-under-addition and fractal structure of markets: Implications for the investment management industry and emphasized examination of matif notional contract, Math. Comput. Model. 29, 37 (1999).

${ }^{43} \mathrm{G}$. Tammann and W. Hesse, Die abhngigkeit der viscositt von der temperatur bie unterkhlten flssigkeiten, Z. Anorg. Allg. Chem. 159, 245 (1926).

${ }^{44} \mathrm{H}$. Vogel, The law of the relationship between viscosity of liquids and the temperature, Phys. Z. Leipz. 22, 645 (1921).

${ }^{45} \mathrm{G}$. S. Fulcher, Analysis of recent measurements of the viscosity of glasses, J. Am. Ceram. Soc. 8, 339 (1925).

${ }^{46}$ P. Frübing, D. Blischke, R. Gerhard-Multhaupt and M. S. Khalil, Complete relaxation map of polyethylene: Filler-induced chemical modifications as dielectric probes, J. Phys. D, Appl. Phys. 34, 3051 (2001).

${ }^{47}$ M. Arndt, R. Stannarius, H. Groothues, E. Hempel and F. Kremer, Length scale of cooperativity in the dynamic glass transition, Phys. Rev. Lett. 79, 2077 (1997).

${ }^{48}$ J. Zimeri and J. Kokini, The effect of moisture content on the crystallinity and glass transition temperature of inulin, Carbohydr. Polym. 48, 299 (2002).

${ }^{49}$ E. Tuncer, Dielectric relaxation and dielectric mixtures: Similarities, Conf. Electrical Insulation and Dielectric Phenomena (CEIDP'05) Annual Report (2005), pp. 725-726.

${ }^{50}$ E. Tuncer, Analogy between dielectric relation and dielectric mixtures: Application of the spectral density representation, J. Phys., Condens. Matter 17, L125 (2005).

${ }^{51}$ E. Tuncer, Structure/property relationship in dielectric mixtures: Application of the spectral density theory, J. Phys. D, Appl. Phys. 38, 223 (2005).

${ }^{52}$ E. Tuncer, Spectral density representation of dielectric mixtures, Appl. Phys. A 107, 575 (2012).

${ }^{53}$ E. Tuncer, Geometrical description in binary composites and spectral density representation, Materials 3, 585 (2010).

${ }^{54} \mathrm{E}$. Tuncer, Extracting spectral density function of a binary composite without a priori assumption, Phys. Rev. B 71, 012101 (2005).

${ }^{55} \mathrm{R}$. Fuchs and F. Claro, Spectral representation for the polarizability of a collection of dielectric spheres, Phys. Rev. B 39, 3875 (1989).

${ }^{56}$ O. Wiener, Die Theorie des Mischkörpers für das Feld der statonären Strömung I. Die Mittelwertsätze für Kraft, Polarisation und Energie, Abh. Math. Phys. Kl. Königl, Sächs. Ges. Wiss. 32, 509 (1912).

${ }^{57}$ G. W. Milton, D. J. Eyre and J. V. Mantese, Finite frequency range kramers kronig relations: Bounds on the dispersion, Phys. Rev. Lett. 79, 3062 (1997). 
${ }^{58}$ R. Sawicz and K. Golden, Bounds on the complex permittivity of matrix particle composites, J. Appl. Phys. 78, 7240 (1995).

${ }^{59} \mathrm{~J}$. Helsing, Bounds on the conductivity of some two-component composites, J. Appl. Phys. 73, 1240 (1993).

${ }^{60} \mathrm{~K}$. Golden and G. Papanicolaou, Bounds for effective parameters of multicomponent media by analytical continuation, J. Stat. Phys. 40, 655 (1985).

${ }^{61} \mathrm{~K}$. Golden, Bounds on the complex permittivity of a multicomponent material, J. Mech. Phys. Solids 34, 333 (1986).

${ }^{62}$ D. J. Bergman, Analytical properties of the complex effective dielectric constant of a composite medium with application to the derivation of rigorous bounds and to percolation problems, Electrical Transport and Optical Properties of Inhomogeneous Media, eds. J. C. Garland and D. B. Tanner, AIP Conference Proceedings, Vol. 40 (American Institute of Physics, New York, 1984), pp. 46-62.

${ }^{63}$ D. J. Bergman, Rigorous bounds for complex dielectric constant of a two-component composite, Ann. Phys. 138, 78 (1982).

${ }^{64} \mathrm{G}$. W. Milton, Bounds on the complex permittivity of a twocomponent composite material, J. Appl. Phys. 52, 5286 (1981).

${ }^{65} \mathrm{G}$. W. Milton, Bounds on the transport and optical properties of a two-component composite material, J. Appl. Phys. 52, 5294 (1981).

${ }^{66} \mathrm{G}$. W. Milton, Bounds on the electromagnetic, elastic, and other properties of two-component composites, Phys. Rev. Lett. 46, 542 (1981).

${ }^{67}$ D. K. Hale, The physical properties of composite materials, J. Mater. Sci. 11, 2105 (1976).

${ }^{68}$ D. J. Bergman, Dielectric constant of a two-component granular composite: A practical scheme for calculating the pole spectrum, Phys. Rev. B 19, 2359 (1979).

${ }^{69} \mathrm{G}$. E. Archie, The electrical resistivity $\log$ as an aid in determining some reservoir characteristics, Trans. Amer. Inst. Min. Metall. Eng. 146, 54 (1942).
${ }^{70}$ S. P. Friedman, Soil properties influencing apparent electrical conductivity: A review, Comput. Electron. Agric. 46, 45 (2005).

${ }^{71}$ S. P. Friedman, A saturation degree-dependent composite spheres model for describing the effective dielectric constant of unsaturated porous media, Water Resour. Res. 34, 2949 (1998).

${ }^{72}$ P. Sheng, Effective-medium theory of sedimentary rocks, Phys. Rev. B 41, 4507 (1990).

${ }^{73}$ R. Hilfer, Local-porosity theory for flow in porous media, Phys. Rev. B 45, 7115 (1992).

${ }^{74}$ B. Nettelblad and G. A. Niklasson, Dielectric relaxations in liquidimpregnated porous solids, J. Mater. Sci. 32, 3783 (1997).

${ }^{75}$ B. Nettelblad, B. Zhu and B.-E. Mellander, Interfacial conduction in ionically conducting two-phase materials: Calculations using the grain consolidation model, Phys. Rev. B 55, 6232 (1997).

${ }^{76} \mathrm{E}$. Tuncer and M. Wegener, Elastic properties of highly anistropic thin poly(propylene) foams, Mater. Lett. 58, 2815 (2004).

${ }^{77}$ E. Tuncer and S. B. Lang, Numerical extraction of distributions of space-charge and polarization from laser intensity modulation method, Appl. Phys. Lett. 86, 071107 (2005).

${ }^{78}$ J. R. Macdonald and E. Tuncer, Deconvolution of immittance data: Some old and new methods, J. Elctroanal. Chem. 602, 255 (2007).

${ }^{79}$ E. Tuncer and J. R. Macdonald, Comparison of methods for estimating continuous distributions of relaxation times, J. Appl. Phys. 99, 074106 (2006).

${ }^{80}$ E. Tuncer and S. M. Gubański, On dielectric data analysis: Introduction of the Monte Carlo method to obtain distributions of relaxation times and a comparison with a functional approach, IEEE Trans. Dielectr. Electr. Insul. 8, 310 (2001).

${ }^{81}$ I. Fredholm, Sur une nouvelle méthode pour la résolution du probléme de dirichlet, Kong. Vetensk.-Akad. Förh 57, 39 (1900). 